\title{
Youssef syndrome- manifesting as cyclical hematuria during non- pregnant state and as antepartum hemorrhage during pregnant state
}

\section{Arun Ramakrishnarao Mahale*, Pankaj Narayan Baravkar}

\author{
Department of Obstetrics \& Gynaecology, MIMSR Medical College, Latur, Maharashtra, India
}

Received: 26 August 2015

Revised: 09 September 2015

Accepted: 10 September 2015

*Correspondence:

Dr. Arun R. Mahale,

E-mail: mahales@hotmail.com

Copyright: (C) the author(s), publisher and licensee Medip Academy. This is an open-access article distributed under the terms of the Creative Commons Attribution Non-Commercial License, which permits unrestricted non-commercial use, distribution, and reproduction in any medium, provided the original work is properly cited.

\begin{abstract}
A woman developed an utero-vesical fistula following her second cesarean section. Between pregnancies she manifested cyclical menouria without urinary incontinence. In her third pregnancy for first 3 months her cyclical menouria was relieved as a feature of pregnancy amenorrhea, but it recurred in fourth month of pregnancy as antepartum hemorrhage because of placental location in the fistula area.
\end{abstract}

Keywords: Vesical fistula, Fistula menstrual, Antepartum haemorrhage, Youssef syndrome

\section{INTRODUCTION}

Fistula is described as per the substance it transfers. Urinary fistulae transfer urine. Supra-isthmic uterovesical fistula is a menstrual fistula, as it transports menstrual blood from uterus to the bladder (menouria). This report relates to such a fistula which manifested cyclical hematuria during non-pregnant state and antepartum hemorrhage in hematuria form during pregnant state. In fact, it is a case of Youssef Syndrome but with an un-described presentation during pregnant state. ${ }^{1}$

\section{CASE REPORT}

A 30 year lady presented with cyclical hematuria every month for 4 years. She did not get menstruation in the form of vaginal bleeding in these 4 years. She also complained of burning in micturition for the last 5 months.

She noted her first bout of hematuria 45 days after her second cesarean section. This cesarean section was done for prolonged labour in the year 2007. Following this she continued to have hematuria till she again became pregnant in the year 2010. During this pregnancy at 4 months gestation she again developed hematuria (while still pregnant) which was continuous not stopping on its own. Ultrasonography revealed low lying placenta. Thus in fact this hematuria during pregnancy was placental site bleeding (antepartum hemorrhage) escaping into the bladder. For this problem her pregnancy was terminated by hysterotomy at a tertiary centre. Next month, after hysterotomy, she again started getting cyclical hematuria. Her hematuria always lasted for 4 to 5 days. She approached us for relief of hematuria, relief from urinary burning, and also requested tubal ligation.

She was para 2 (both by cesarean section), 1 hysterotomy, 2 living and was regularly menstruating but in the form of hematuria. Other than routine examinations this patient was subjected to abdominal and endovaginal sonography, methylene blue test and cystoscopy. Methylene blue test revealed no vaginal leakage of dye even after walking. She micturated out all methylene blue at the termination if test. On cystoscopy a bladder stone 
was seen at the fistula site on the posterior wall of bladder. The catheter specimen of her urine, during menstrual phase (hematuria), was haemorrhagic. It was photographed (Figure-1). Thus this patient manifested menouria for 4 years. Her bleeding of threatened abortion or antepartum hemorrhage also manifested through bladder (urine) and not through vagina. Her amenorrhea cannot be called as true amenorrhea; it was in fact false amenorrhea. This woman menstruated every month in to the bladder through the fistulous opening which communicated the uterine cavity to the bladder. The opening on the lower anterior wall of the uterus which communicated to the bladder cavity, through opening on the posterior wall of bladder, is obvious in the photograph of the hysterectomy specimen (Figure-2).

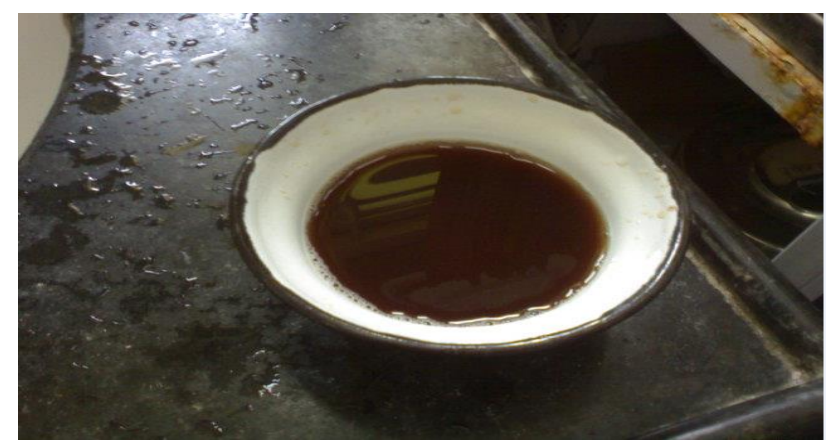

Figure 1: Blood stained urine (menouria).

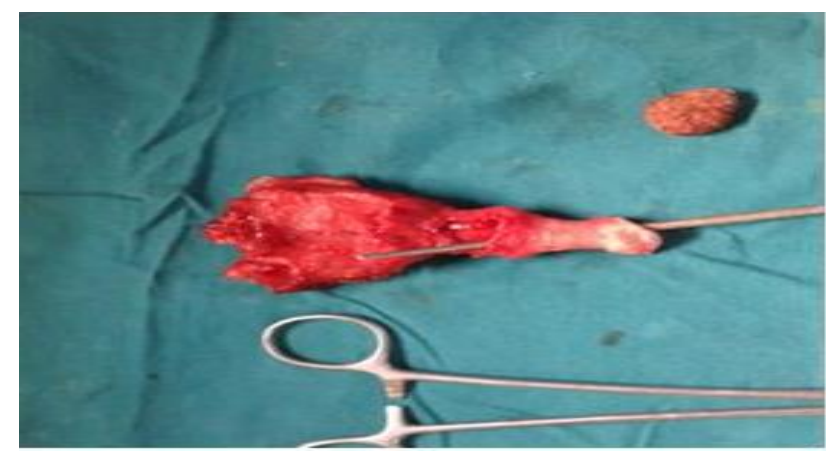

Figure 2: Uterine part of utero-vesical fistula in supra-isthmic portion of uterus and bladder stone.

The patient was subjected to laparotomy. The anterior surface of the uterus was adherent to the anterior wall of the abdomen along its entire length (post-cesarean section uterine ventro-fixation). This was dissected and uterovesical fold area (which was not clearly obvious in this case because of fibrosis) was reached. Bladder was dissected away from the uterus. An opening in the bladder wall in supratrigonal area became obvious. A stone was removed through fistula that is seen in photograph (Figure-2). The anterior surface of uterus appeared mutilated and it was decided to remove the uterus. Hysterectomy was done; the bladder opening was repaired in two layers. Patient was catheterized for two weeks. Patient micturated well and methylene blue test revealed no vesico-vaginal fistula on day 14 . She went home continent and satisfied.

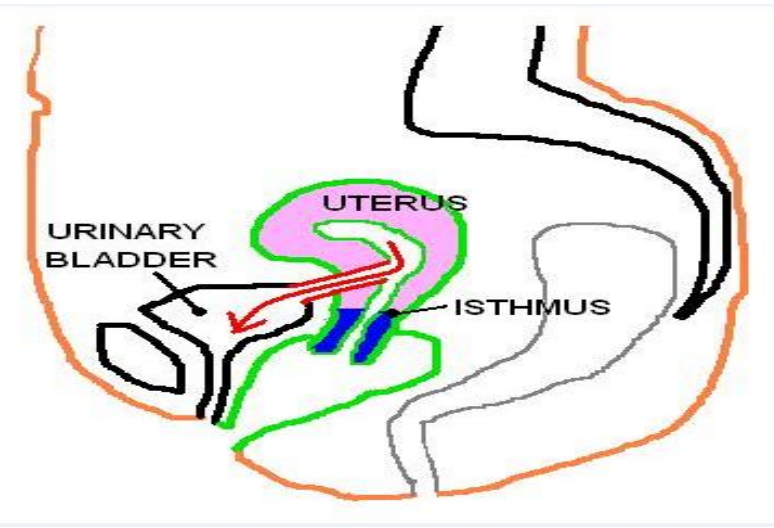

Figure 3: The supra-isthmic position of fistula, and the mechanism of menouria (menstrual fistula).

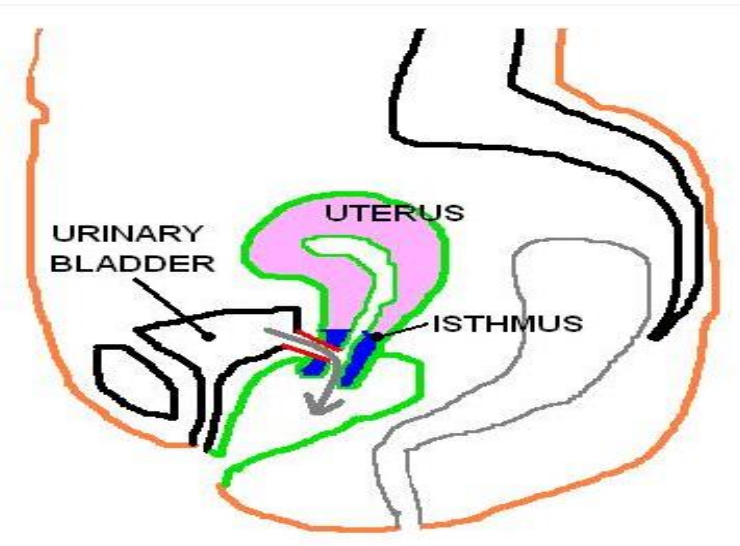

Figure 4: The infra-isthmic position of fistula and the mechanism of urinary incontinence (urinary fistula).

\section{DISCUSSION}

The most common form of urogenital fistula is vesicovaginal fistula. Fistulae communicating bladder and uterus are rare type of urogenital fistulae representing about $1 \%$ to $4 \%$ of all urogenital fistulae. Most uterusbladder fistulae follow a complicated caesarean delivery. Inadequate mobilization of bladder inferiorly or laterally, bladder injury while delivering a large fetal head or accidental inclusion of bladder wall in to the uterine suturing are the reasons. Other possible causes include difficult vaginal delivery, rupture of lower uterine segment \& bladder due to traumatic forceps delivery, migration of an intrauterine contraceptive device and very rarely due to necrosis of bladder wall directly over dehiscence of lower segment caesarean section scar. Malignancy is another cause.

The presentation of utero-vesical fistula largely depends on the level of the fistula. Fistulous opening above the 
isthmus causes different manifestations than when it is below the isthmus. Bladder is a low pressure distensible organ. With increasing urinary filling the bladder relaxes and prevents rise in intravesical pressure. As against this uterus is a thick muscular organ that resists acute distension. It contracts vigorously even with slight intrauterine collections. Cervix is fibrous with relatively little muscular tissue. Opening the cervical canal requires great force which uterine musculature generates by strong contractions during menstruation, miscarriage and labour. Presence of a fistula above the isthmus creates a communication between high pressure uterine cavity and low pressure bladder cavity. This tract opens very easily with slight rise in intrauterine pressure during menstruation. The leakage of intrauterine pressure into the bladder prevents its further rise and fails to open the cervical canal. Thus supra-isthmic fistulae always leak uterine contents into the bladder (Figure-3). Bladder being a low pressure system fails to push its contents into the uterine cavity through supra-isthmic fistulous tracts. Thus supra-isthmic fistulous tracts rarely cause urinary leakage into the genital tract. The result is 'Youssef Syndrome'-that is, amenorrhea (absence of vaginal menstrual bleeding), periodic hematuria (termed 'menouria' by Youssef) and absence of urinary leakage through vagina. ${ }^{1}$ Such cases, which do not leak urine but divert menstrual blood, should in fact be labelled as menstrual fistula instead of urinary fistula.

When the fistulous tract is below the isthmus, it is in fact a vesico-cervical fistula, it results into true urinary incontinence and no menouria (Figure-4).

The case presented here also had other unusual presentations. She conceived in spite of uterovesical fistula. In this pregnancy she had low implantation of placenta causing symptoms of antepartum hemorrhage. But this antepartum hemorrhage was not in the form of vaginal bleeding but urinary bleeding. For which she was subjected to hysterotomy (pregnancy termination).
Surgery is the main stay \& definitive treatment of uterovesical fistula, although spontaneous healing occurs in $5 \%$ of cases. ${ }^{2}$ Although few small fistulas have been reported to close either spontaneously or through cystoscopic fulguration, most of them require surgery. Fistulas detected within 48 hrs. should be repaired early, while fistulas reporting later than this should be repaired after 2 to 3 months, allowing tissues to subside oedema and inflammation. Fistula repair should be approached trans-abdominally in cases of large fistula. Omental flap interposition aids in successful fistula repair. Hysterectomy may be omitted in the presence of very small uterine defect but it may be done if indicated for other reasons including the presence of a large uterine defect.

\section{ACKNOWLEDGEMENT}

The authors are thankful to Dean, MIMSR Medical College, Latur for permitting us to publish this case report.

\section{Funding: No funding sources \\ Conflict of interest: None declared \\ Ethical approval: Not required}

\section{REFERENCES}

1. Youssef AF. Menouria following lower segment cesarean section: A syndrome. Am J Obstet Gynecol. 1957;73:759-67.

2. Porcaro AB, Zicari M, Zecchini Antoniolli S, Pianon $\mathrm{R}$, Monaco C, Migliorini F, et al. Vesicouterine fistulae following caesarean section: report on a case, review and update of the literature. Int Urol Nephrol. 2002:34(3):335-44.

Cite this article as: Mahale AR, Baravkar PN.

Youssef syndrome- manifesting as cyclical hematuria during non-pregnant state and as antepartum hemorrhage during pregnant state. Int J Reprod Contracept Obstet Gynecol 2015;4:1663-5. 